\title{
Synthetic biology: below the radar
}

Book

Reviewed by

Abstract

Keywords
EngelHard, M. ed. (2016). Synthetic Biology Analysed: Tools for

Discussion AND EVALUATION. SPRINGER INTERNATIONAL.

\section{Emma Weitkamp}

Englehard et al provide a wide-ranging look at synthetic biology, from discussion of how one might classify different synthetic approaches to consideration of risk and ethical issues. The chapter on public engagement considers why synthetic biology seems to sit below the public radar.

Public engagement with science and technology; Public perception of science and technology; Risk communication

How will synthetic biology shape our conceptions of life? This is just one of the many 'big' questions raised in this collection of readings aimed at stimulating discussion and providing an 'interdisciplinary toolbox to facilitate and differentiate its societal evaluation' (p. vii). The book provides an interesting exploration of the manifold social, ethical and legal issues raised by modern technology. Yes it focuses on synthetic biology, but the issues raised apply to varying degrees to a wide range of emerging technologies, such as robotics and nanotechnology.

What makes synthetic biology particularly interesting, though, is its potential to change radically how we think about life, the questions it raises about risk analysis and its, perhaps surprisingly low, public salience. Taking the ethical questions first, Christian Illies, in the chapter New Debates in Old Ethical Skins asks whether synthetic biology demands a new ethics. Having worked through issues related to risk and uncertainty, global justice and the 'playing God' argument Illies considers that at least at the moment 'most challenges of synthetic biology are familiar in principle... They do not require a new ethics if 'new ethics' is understood as a field of new norms and values.' (p. 110) But he does go on to suggest that synthetic biology, as the 'apogee of modern science and technology... might also be regarded as an extreme case of uncertainty.' (p.110) As such, it does warrant ethical debate and Illies calls for this debate to happen before 'before the developments of synthetic biology urgently demand ethical or political answers'. (p.122).

Synthetic Biology Analysed is not an easy read and the book would certainly have benefited from the services of a good editor. Part of the challenge is the wide range of literature and research fields and traditions represented in the text. Not only does it seek to explain the range of organisms we might classify as synthetic (and how we might classify them), but also how we might develop an ethical framework 
to address synthetic biology, the types and nature of the risks that should be considered, current public opinion research and what sort of legal framework we might imagine for this emerging field. As such, it is a comprehensive introduction to the subject.

Bölker, Engelhard and Budisa provide a useful way of conceptualising the different strands of synthetic biology in chapter 2, Synthetic Biology: Diverse Layers of Life. They describe three, increasingly challenging, branches of synthetic biology:

- Engineering approaches: in this conception, biology is transformed into an engineering discipline, with standardised parts and building blocks (see for example: biobricks.org) used to modify existing life forms (and presumably potentially to build a new life form from scratch). Engineering approaches stick fairly closely to natural systems; this presents both a risk - in that any organisms created can potentially cross bread with natural organisms - and a suggestion for risk assessment - in that we can use our knowledge of natural systems as a guide.

- Orthogonal approaches: these approaches seek to reduce risk by creating organisms unable cross breed with natural life forms, by introducing, for example, synthetic nucleotides. The use of synthetic nucleotides creates, what is referred to as 'xeno-life'. Of course, such xeno-organisms could still compete with natural organisms in the ecosystem, so are not without risk.

- Protocell research: drawing on evolutionary biology, protocell research seeks to create simple cells that can perform at least some of the functions associated with life (specifically metabolic functions). Protocell research is often aimed at exploring the origins of life, but questions around risk remain.

Perhaps the most interesting and challenging chapter is that from Engelhard, Bölker and Budisa exploring Old and New Risks in Synthetic Biology: Topics and Tools for Discussion. The chapter explores whether our existing risk management systems can be applied to synthetic biology and arguing that a multidisciplinary approach to risk assessment, that takes account of the precautionary principle, is needed. The chapter highlights the important role of perspective in shaping risk discourses around synthetic biology: is it novel (i.e. risky) or just a continuation of existing genetic research (i.e. lower risk). How the field presents its identity could have a significant impact on how risks are assessed, particularly by nonspecialists. The authors also highlight features of synthetic biology that present particular challenges for risk assessment:

- the depth of the intervention: the authors argue that synthetic biology, by seeking to add new biological parts to systems, seeks a greater depth of modification of organisms than previous technologies, such as genetic engineer.

- Familiarity: as organisms (particularly orthogonal approaches to synthetic biology) diverge from natural organisms, our ability to assess risks based on similarity with natural systems diminishes. Some do argue that more divergent organisms are in fact safer because they cannot directly interact on a genetic level with natural systems. 
When it comes to public perception, however, Pardo Avellaneda and Hagen's chapter Synthetic Biology: Public Perceptions of an Emergent Field, highlights the low salience of the topic with the public. As they note, this is not uncommon. 'Most scientific developments take place silently, contributing to the continuous expansion of knowledge but known only to the corresponding subsets of the scientific community' (p. 135). Synthetic biology is at an early stage of development, it has not yet produced any notable 'products' or practical developments that might capture public attention. The field is complex, presenting barriers to communication, so it is not surprising that it remains relatively infrequently reported. Pardo Avellaneda and Hagen argue that given the recent experience of public (non)acceptance of biotechnology, now is the time to begin a public conversation about synthetic biology. As is apparent from the wide range of issues addressed in this book, a public discussion is needed to ensure that synthetic biology travels in a publicly acceptable direction. The Responsible Research and Innovation (RRI) agenda demands this level of public and policy engagement.

Author

Dr. Emma Weitkamp is Associate Professor of Science Communication at the University of the West of England, Bristol and Editor in Chief of JCOM. Her research focuses on narrative approaches to science communication, both in the media and through the arts. E-mail: emma.weitkamp@uwe.ac.uk. 